\title{
ON THE MAGNON SPECTRUM OF ANTIFERROMAGNETICALLY ALIGNED BILAYER FILMS
}

\author{
H. Puszkarski, B. Kolodziejczak \\ Surface Physics Division, Institute of Physics, A. Mickiewicz University \\ Matejki 48/49, 60-769 Poznani, Poland \\ A. Akjouj, B. Djafari-Rouhani and T. Dobrzynski \\ Laboratoire de Dynamique et Structure des Matériaux Moléculaires \\ Unité Associeé au CNRS 801 \\ Université des Sciences et Techniques de Lille I, 59655 Villeneuve d'Ascq Cedex, France
}

We deal with spin-wave propagation in two antiferromagnetically aligned sublayers forming one bilayer film owing to the antiferromagnetic exchange coupling assumed to exist at the film interface. We get the following picturc: as the spin wave traverses the interface, the creation of a spin-reversal in the one sublayer becomes an annihilation in the other sublayer. This feature is expressed by the non-conventional type of normalization condition of the spin-wave amplitudes.

PACS numbers: 75.70.-i, 75.50.Ee

\section{Introduction}

In the last few years considerable interest has been devoted to the physical properties of magnetic multilayers with a view to their possible applications in various electronic (in particular - memory) devices. One crucial point in the understanding of such systems is the case of antiferromagnetic coupling at the interface between two magnetic layers. This system has been studied earlier for a slab adsorbed on a semi-infinite [1] substrate and also for semi-infinite superlattices [2-5]. A new exciting surface phase transition was, in particular, predicted [2]. Although the magnon spectrum was studied previously in the above system [4], no such work has appeared (to our knowledge) for the more elementary system formed by a bilayer with antiferromagnetic coupling. This system is interesting by itself and has already been approached from the experimental point of view [6-7]. It is also interesting as the elementary brick for more complex composite magnetic systems. We thus shall proceed to a study of the magnon frequencies within the simplest possible model - a bilayer film with antiferromagnetic interfacial coupling. 


\section{The model}

We consider a film consisting of two ferromagnetic materials in the shape of thin layers (sublayers $A$ and $B$ ) of homogeneous structure extending unboundedly in directions parallel to the surface of the film. In general, the two sublayers are assumed to have different magnetic properties; however, for simplicity, we shall assume that their crystallographic structures are identical. The two sublayers form one magnetic system owing to the antiferromagnetic exchange coupling assumed to exist at the interface separating them.

On the above assumptions, atoms lying in the same lattice plane parallel to. the surface (to be termed in brief "a plane") are in identical physical conditions, i.e. they are mutually equivalent. The two sublayer surface planes, which form the interface, will be referred to as the "interface planes". An atom is labelled by an index $\ell j$, where $\ell$ is a number denoting the plane, and $j$ is a two-dimensional vector lying in the plane of the film. As it is shown in Fig. 1, the index $\ell$ takes the following values: $\ell=0$ (surface plane $A$ ) $\ell=1,2, \ldots N-2$ (internal planes $A$ ), $\ell=N-1$ (interface plane $A$ ), $\ell=N$ (interface $B$ ), $\ell=N+1, N+2, \ldots L-2$ (internal planes $B), \ell=L-1$ (surface plane $B)$.

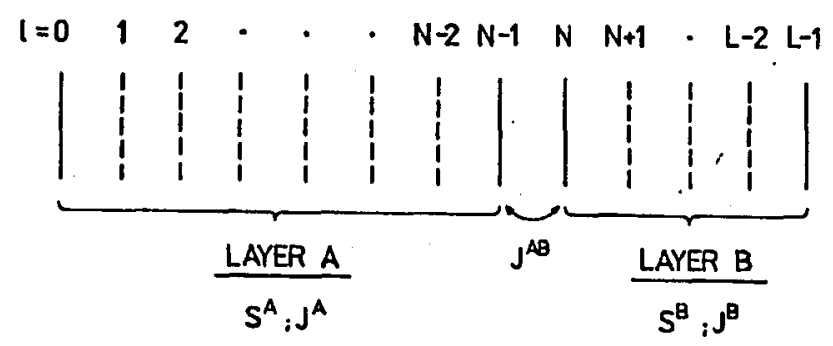

Fig. 1. Model of a bilayer film consisting of two ferromagnetic sublayers $A$ and $B$, coupled together by the interface antiferromagnetic excliange integral $J^{A B} . N$ is the number of lattice planes in sublayer $A$, whereas $L-N$ is their number in sublayer $B$. The ferromagnetic sublayers are assumed to differ with respect to their spin numbers $\left(S^{A}\right.$ and $S^{B}$, respectively) and nearest-neighbour exchange integrals $\left(J^{A}\right.$ and $\left.J^{B}\right)$; the spins $S^{A}$ and $S^{B}$ are aligned antiferromagnetically.

Starting from the static problems we assume, in a semi-classical approximation, that a spin present in a lattice site can be represented as $S_{\ell j}=S^{A} \gamma^{A}$ for sublayer $A$ and $S_{\ell j}=S^{B} \gamma^{B}$ for sublayer $B$, where $S^{A ; B}$ are the respective spin numbers (in $\hbar$ units), and $\boldsymbol{\gamma}^{A ; B}$ denotes a unit vector in the quantization direction shared by all the spins of the sublayer $A$ or $B$, respectively. We chose the system of coordinates in such a way that the $x y$-plane is parallel to the film surface and the $z$-axis is perpendicular thereto. We assume that all the spins are aligned parallel to the film, i.e. $\gamma_{z}^{A ; B} \equiv 0$; consequently, the sublayer magnetization directions are restricted to the $x y$-plane.

In the next Section we shall perform calculations of the dynamical properties; this will be done within the framework of the Heisenberg localized spin model 
assuming exchange (nearest-neighbour) interaction and a Zeeman Hamiltonian in a standard form

$$
\widehat{\mathcal{H}}=-\sum_{\ell j \neq \ell^{\prime} j^{\prime}} J_{\ell \ell^{\prime}} \widehat{S}_{\ell j} \cdot \widehat{S}_{\ell^{\prime} j^{\prime}}-g \mu_{\mathrm{B}} \sum_{j} H \cdot \widehat{S}_{\ell j}
$$

where the first summation extends over pairs of neighbouring spins; the exchange integral $J_{\ell \ell^{\prime}}$, between nearest neighbours situated respectively in planes $\ell$ and $\ell^{\prime}$, is assumed to be $J_{\ell \ell^{\prime}}=J^{A}$ if both interacting spins belong to the sublayer $A$, $J_{\ell \ell^{\prime}}=J^{A B}$ if they belong to different sublayers, and $J_{\ell \ell^{\prime}}=J^{B}$ if they belong to the sublayer $B$. The interface coupling exchange integral is assumed to be of the antiferromagnetic type, i.e. $J^{A B}<0$. The external field $H$ takes any orientation within the $x y$-plane and one assumes that $\boldsymbol{\gamma}^{A}$ and $\boldsymbol{\gamma}^{B}$ are directed, respectively, parallelly and antiparallelly to the direction of that field. To establish the conditions under which such an "antiferromagnetically aligned" (AF) ground state of the bilayer system is stable, we require its energy to be lower than the ground state energy of the respective "ferromagnetically aligned" (FF) bilayer film (for which $\boldsymbol{\gamma}^{A} \equiv \boldsymbol{\gamma}^{B}$ ). This requirement leads to the following stability condition to be satisfied by $H$ and $J^{A B}$ for the AF aligned bilayer film (i.e. for which $\boldsymbol{\gamma}^{B}=-\boldsymbol{\gamma}^{A}$ ):

$$
g \mu_{\mathrm{B}} S^{B} H(N-L) \leq-2 S^{A} S^{B} z_{\perp} J^{A B},
$$

where $z_{\perp}$ denotes the number of nearest neighbours situated in an adjacent plane, and $(N-L)$ is the thickness of the sublayer $B$ (in lattice units). The physical meaning of the inequality (2) is the following: let us note that on its right-hand side we have the absolute value of the energy (calculated per interface spin of the sublayer $B$ ) of the respective exchange coupling through the interface, while the left-hand term represents the Zeeman energy of the spin $S^{B}$, multiplied by the number of atomic planes in the sublayer $B$. Therefore, it follows from the condition (2) that our AF bilayer ground state will be stable if the value of the external field is properly matched to the actual values of the antiferromagnetic interface coupling and the thickness of the sublayer $B$; the total (positive) Zeeman energy of the sublayer $B$ should not exceed the (negative) energy of coupling between the interface spins of the sublayer $B$ and the interface spins of the sublayer $A$.

\section{Spin waves}

We diagonalize the Hamiltonian (1) applying the procedure described in detail in Ref. [8]. From the diagonalization procedure it follows that the spin-wave functions have the following form:

$$
\Psi_{\ell j}\left(k_{\|}, k_{\perp}\right)=u_{\ell}\left(k_{\perp}\right)=u_{\ell}\left(k_{\perp}\right) \exp \left(\mathrm{i} k_{\|} \cdot j\right)
$$

where $k_{\|}$is the wave vector lying in the film plane, and $k_{\perp}$ is a $z$-component of the wave vector in the direction perpendicular to the film plane. While the former quantity is a true wave vector describing propagation in the plane of the film, the quantity $k_{\perp}$ is a quantum number determining the variation of the amplitude $u_{\ell}\left(k_{\perp}\right)$ of the spin-precession cone in the direction perpendicular to the film plane. The spin-wave mode amplitudes, $u_{\ell} \equiv u_{\ell}\left(k_{\perp}\right)$, have to satisfy the appropriate set of $2 L$ homogeneous equations; however, it turns out that, in fact, this set of 
equations consists of two, mutually independent sets of $L$ equations. Let us write explicitly the first set of the two

$$
\begin{array}{ll}
{[x(-E)-a] u_{0}-u_{1}=0,} & \ell=0, \\
-u_{\ell-1}+x(-E) u_{\ell}-u_{\ell+1}=0, & \ell=1,2, \ldots N-2, \\
-u_{N-2}+[x(-E)-b] u_{N-1}-\varepsilon u_{N}=0, & \ell=N-1, \\
-\varepsilon^{\prime} u_{N-1}+[y(+E)-c] u_{N}-u_{N+1}=0, & \ell=N, \\
-u_{\ell-1}+y(+E) u_{\ell}-u_{\ell+1}=0, & \ell=N+1, \ldots L-2, \\
-u_{L-2}+[y(+E)-d] u_{L-1}=0, & \ell=L-1,
\end{array} .
$$

where we have introduced the following notations:

$$
\begin{aligned}
& x(\mp E)=\mp \frac{E}{2 S^{A} J^{A} \Gamma_{\perp}}+\frac{1}{\Gamma_{\perp}}\left[\left(z_{\|}+2 z_{\perp}-\Gamma_{\|}\right)+\frac{g \mu_{\mathrm{B}} H}{2 S^{A} J^{A}}\right], \\
& y( \pm E)= \pm \frac{E}{2 S^{B} J^{B} \Gamma_{\perp}}+\frac{1}{\Gamma_{\perp}}\left[\left(z_{\|}+2 z_{\perp}-\Gamma_{\|}\right)+\frac{g \mu_{\mathrm{B}} H}{2 S^{B} J^{B}}\right], \\
& a=d=z_{\perp} / \Gamma_{\perp} \\
& b=\frac{z_{\perp}}{\Gamma_{\perp}}\left(1+\frac{S^{B} J^{A B}}{S^{A} J^{A}}\right), \quad \quad c=\frac{z_{\perp}}{\Gamma_{\perp}}\left(1+\frac{S^{A} J^{A B}}{S^{B} J^{B}}\right), \\
& \varepsilon=\sqrt{\frac{S^{B}}{S^{A}}} \frac{J^{A B}}{J^{A}}, \quad \varepsilon^{\prime}=\sqrt{\frac{S^{A}}{S^{B}}} \frac{J^{A B}}{J^{B}} .
\end{aligned}
$$

The second set of equations is to be obtained from Eqs. (4a) by replacing the eigenenergies $E$ (present in all the diagonal terms) by " $-E$ ":

$$
\begin{array}{ll}
{[x(+E)-a] u_{0}-u_{1}=0,} & \ell=0, \\
-u_{\ell-1}+x(+E) u_{\ell}-u_{\ell+1}=0, & \ell=1,2, \ldots N-2, \\
-u_{N-2}+[x(+E)-b] u_{N-1}-\varepsilon u_{N}=0, & \ell=N-1, \\
-\varepsilon^{\prime} u_{N-1}+[y(-E)-c] u_{N}-u_{N+1}=0, & \ell=N, \\
-u_{\ell-1}+y(-E) u_{\ell}-u_{\ell+1}=0, & \ell=N+1, \ldots L-2, \\
-u_{L-2}+[y(-E)-d] u_{L-1}=0, & \ell=L-1 .
\end{array}
$$

Within the framework of our model, only such orientations of the film surface can be considered at which the nearest neighbours of a given spin belong to the same plane and the two closest lying planes. In cubic lattices, the orientations of this kind occurring most commonly in practice are: $\operatorname{sc}(001), \mathrm{fcc}(001)$ and $\mathrm{bcc}(001)$. The relevant structural factors are defined as follows:

$$
\Gamma_{\|}=\sum_{\delta_{\|}} \exp \left[\mathrm{i}\left(k_{\|} \cdot \delta_{\|}\right)\right] \quad \text { and } \quad \Gamma_{\perp}=\sum_{\delta_{\perp}} \exp \left[\mathrm{i}\left(k_{\|} \cdot \delta_{\perp}\right)\right]
$$

where $\delta_{\|}$denotes any vector connecting a site in a plane $\ell$ with its $z_{\|}$nearest neighbours in the same plane, and $\delta_{\perp}$ denotes the projection onto the $x y$-plane of any vector connecting a site in the plane $\ell$ with its $z_{\perp}$ nearest neighbours in the plane $\ell+1$. In Table the structural factors for all the surface orientations considered here are assembled (the choice of respective $k_{1}, k_{2}$ coordinates is shown in Fig. 2).

The two sets (4a) and (4b) obviously form a set of $2 L$ equations with $2 L$ unknowns, notwithstanding the fact that the problem under consideration is 


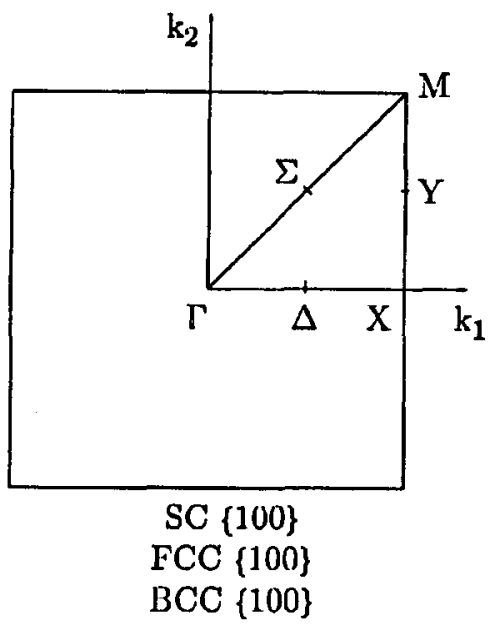

Fig. 2. The 1st Brillouin zone for (001) cubic structures with the characteristic points of the zone indicated thereon.

TABLE

Structural factors for (001) surface orientations.

\begin{tabular}{c|c|c|c|c}
\hline \hline & $z_{\|}$ & $z_{\perp}$ & $\Gamma_{\|}$ & $\Gamma_{\perp}$ \\
\hline SC (001) & 4 & 1 & $2\left(\cos k_{1}+\cos k_{2}\right)$ & 1 \\
FCC (001) & 4 & 4 & $2\left(\cos k_{1}+\cos k_{2}\right)$ & $4 \cos \left(\frac{1}{2} k_{1}\right) \cos \left(\frac{1}{2} k_{2}\right)$ \\
BCC (001) & 0 & 4 & 0 & $4 \cos \left(\frac{1}{2} k_{1}\right) \cos \left(\frac{1}{2} k_{2}\right)$
\end{tabular}

$L$-dimensional. Consequently, while solving these sets of equations one obtains $2 L$ cigenvalues of $E$ which can be ordered in $L$ pairs consisting of one positive and one respective negative value: $E$ and $-E$. In the spin-wave formalism adopted here, the quantity $E$ is the energy of a given spin-wave mode. Of course, physically meaningful solutions correspond to positive $E$ values only, since the requirement of positive $E$ is equivalent to considering elementary excitations of positive energies only. In this way we satisfy the requirement that the selected ground state shall be a state of stable equilibrium of the system. The two sets of solutions obtained (i.e. corresponding to positive eigenenergies and negative ones, respectively) cannot simultaneously fulfill the same normalization conditions. Thus, the normalization conditions read (see [8])

$$
\sum_{\ell=0}^{N-1}\left|u_{\ell}\right|^{2}-\sum_{\ell=N}^{L-1}\left|u_{\ell}\right|^{2}= \pm 1,
$$

where +1 corresponds to solutions of the set (4a) and -1 to solutions of $(4 \mathrm{~b})$, or vice versa.

To characterize the spin-wave modes of a bilayer film it is necessary to in- 
troduce two perpendicular wave vector components, namely $k_{\perp}^{A}$ and $k_{\perp}^{B}$, each of the two being assigned to one particular sublayer. They are to be defined by the following equalities:

$$
x( \pm E) \equiv 2 \cos \left(k_{\|}^{A}\right), \quad y( \pm E) \equiv 2 \cos \left(k_{\perp}^{B}\right) .
$$

This allows us to express the solutions $u_{\ell}$ in terms of the respective wave numbers $k_{\perp}^{A}$ and $k_{\perp}^{B}$

$$
u_{\ell}= \begin{cases}u_{\ell}\left(k_{\perp}^{A}\right) & \text { for } \ell \in \text { sublayer } A \\ u_{\ell}\left(k_{\perp}^{B}\right) & \text { for } \ell \in \text { sublayer } B .\end{cases}
$$

The respective spin-wave mode functions can be found by applying one of the following three different approaches: the recurrent interface rescaling approach $[9,10]$, the interface response-rescaling approach [11], or the transfer matrix approach [12].

By means of Eqs. (8) and (5), the spin-wave energy $E$ can be expressed by either of the perpendicular wave numbers introduced above. One easily finds that there exist four energetical branches, but only two of them correspond to physical solutions with $E>0$; these are as follows:

$$
\begin{aligned}
& E_{1}=4 S^{A} J^{A}\left[z_{\perp}-\Gamma_{\perp} \cos \left(k_{\perp}^{A}\right)+2 S^{A} J^{A}\left(z_{\|}-\Gamma_{\|}\right)+g \mu_{\mathrm{B}} H,\right. \\
& E_{2}=4 S^{B} J^{B}\left[z_{\perp}-\Gamma_{\perp} \cos \left(k_{\perp}^{B}\right)+2 S^{B} J^{B}\left(z_{\|}-\Gamma_{\|}\right)+g \mu_{\mathrm{B}} H .\right.
\end{aligned}
$$

The higher branch $E_{1}$ corresponds to the excitation of a spin wave, which originates in the reversal of a spin at a given node of the spin sublattice $A$, whereas the lower branch $E_{2}$ corresponds to the collectivized spin reversal originating in the sublattice $B$.

\section{Conclusions}

The above derived formulae for the spin-wave energies permit the following interpretation: the set of equations (4a) describes the collective propagation of spin reversal produced in the sublattice $A$ (as indicated by the energy term $+g \mu_{\mathrm{B}} H$ ); whereas inversely, the set of equations (4b) describes the propagation of spin reversals produced in the sublattice $B$. We should draw attention to the following, hitherto undiscussed feature of the propagation of the spin-wave process in antiferromagnetically aligned bilayer films, namely: a collective event of creation of a spin reversal in a given sublattice becomes - when it reaches the other sublattice - a collective process of annihilation of an opposite spin reversal. Thus, the propagation of a spin wave in an AF bilayer is in fact a very remarkable process; in the one sublattice it causes an increase in the $z$-component of the spin, whereas it causes a decrease of the $z$-component in the other. This fact is expressed by the non-conventional type of normalization condition (Eq. (7)) we had to apply to the solutions of the sets $(4 a, b)$.

\section{Acknowledgments}

One of the authors (IIP) wishes to express his gratitude to the Laboratoire de Dynamique et Structure des Matériaux Moléculaires (Unité de Recherche Associée 
au C.N.R.S. no. 801), Universite de Lille I for providing the necessary support for his stay in France. Thanks are also due to the Polish Committee for Scientific Research for its support under the grant No. 223619102.

\section{References}

[1] R.E. Camley, Phys. Rev. B 35, 3608 (1987).

[2] T.G. LePage, R.E. Camley, Phys. Rev. Lett. 65, 1152 (1990).

[3] R.E. Camley, D.R. Tilley, Phys. Rev. B 37, 3413 (1988).

[4] T.G. LePage, R.E. Camley, Phys. Rev. B 40, 9113 (1989).

[5] R.E. Camley, Phys. Rev. B 39, 12316 (1989).

[6] P. Grünberg, R. Schreiber, Y. Pang, M.B. Brodsky, H. Sowers, Phys. Rev. Lelt. 57, $2442(1986)$.

[7] C. Carbone, S.F. Alverado, Phys. Rev. B 36, 2433 (1987).

[8] H. Puszkarski, Acta Phys. Pol. A 39, 597 (1971).

[9] H. Puszkarski, Acta Phys. Pol. A 74, 701 (1988).

[10] H. Puszkarski, Solid St. Commun. 72, 887 (1989).

[11] H. Puszkarski, L. Dobrzynski, Phys. Rev. B 39, 1819 (1989).

[12] J.C.S. Lévy, H. Puszkarski, J. Phys., Condens. Malter 3, 5247 (1991). 